\title{
Sprachliche Herausforderungen an die Rechtsvergleichung im Agrarrecht
}

\section{Die agrarrechtliche Fachsprache als besondere Herausforderung}

Die Rechtsvergleichung im Agrarrecht hat in den letzten Jahrzehnten eine wachsende Rolle in der modernen Agrarrechtswirklichkeit eingenommen und ist heute für die agrarrechtliche Forschung unverzichtbar, um das untersuchte Rechtsinstitut umfassend oder innovativ zu behandeln. Professor Roman Budzinowski lebt in besonderem Maße diese Rechtsvergleichung im Agrarrecht. Ein besonderer Schwerpunkt seiner rechtsvergleichenden Forschung ist das italienische Recht. Bereits 1987/88 hat Professor Budzinowski einen Forschungsaufenthalt am Istituto di Diritto Agrario Internazionale e Comparato in Florenz, dem sich in den Folgejahren weitere Aufenthalte am Istituto Europeo in Florenz (Fiesole) und der Università degli Studi di Udine anschlossen. Er hielt Vorlesungen für Studenten in Perugia, Siena, Pisa und Udine. Mit der Universität Macerata verbindet ihn eine langanhaltende Kooperation. Daneben hat Professor Budzinowski auch viel zum deutschen und zum spanischen Recht gearbeitet und hierbei nachhaltige Forschungskooperationen mit insbesondere den Universitäten Göttingen und Frankfurt (Oder) sowie Jaén aufgebaut, die über Generationen von Akademikern fortbestehen. Man kann daher zusammenfassen: Wie kaum ein anderer Agrarrechtler in Europa verkörpert Professor Budzinowski die Rechtsvergleichung im Agrarrecht.

\footnotetext{
* Georg-August-Universität Göttingen, Deutschland.
} 
Vor diesem Hintergrund ist eine Auseinandersetzung mit den Herausforderungen der Rechtsvergleichung im Agrarrecht angebracht. Dabei soll der Fokus gerichtet werden auf ein zentrale Perspektive, die Sprache.

Warum die Sprache? Zunächst ist die Sprache das Werkzeug des Juristen. Die Rechtswissenschaft ist auch eine Kommunikationswissenschaft, da sie notwendigerweise Lebenssachverhalte in Sprache präzise beschreiben und definieren muss. Dabei bedient sich der Jurist neben der lebendigen Sprache vor allem einer Fachsprache. Innerhalb der Wissenschaft haben die einzelnen Rechtsgebiete eine jeweilige Fachsprache entwickelt. So versteht ein Zivilrechtler den Begriff der Gesellschaft anders als ein Verfassungsrechtler. Auch das Agrarrecht hat eine eigene Fachsprache entwickelt. Diese zeichnet sich wie das Agrarrecht selbst dadurch aus, dass sie sehr oft an tradierten Begriffen anknüpft, die kulturell und historisch mit einer bestimmten Region verbunden sind und daher im besonderem Maße als relativ bewertet werden müssen. Ein Beispiel ist der Begriff „Hof“ im deutschen Recht, der an eine tradierte Betriebsstruktur, den landwirtschaftlichen Familienbetrieb anknüpft. Vergleichbare Begriffe wie Farm oder Plantage treffen diese Besonderheit nicht. Ebenso ist der rechtliche Fachbegriff des „Wiederanspannens des Hofs" ein Begriff, der ein Bild (das Anspannen des Pferdes an einen Wagen) aus einer Zeit der Pferdekutschen verwendet, um die Wiederaufnahme eines stillgelegtem Begriffs zu beschreiben. Das Agrarrecht verwendet daher in besonderem Maße eine Sprache, die in besonderem Maße in ein bestimmtes historisches, kulturelles und regionales Umfeld gebettet ist. Der Vergleich ausländischer Regeln des Agrarrechts setzt daher beim Rechtsvergleicher nicht nur Sprachfertigkeiten voraus, sondern auch entsprechende Kenntnisse des historischen, kulturellen und regionalen Umfelds.

\section{Die Europäisierung der Sprache des Agrarrechts}

Eine weitere Besonderheit des Agrarrechts ist die Europäisierung der Sprache. Die Gemeinsame Agrarpolitik der EU hat sich in den letzten 60 Jahren zu dem EU-Politikbereich mit der stärksten Integration entwickelt. Dadurch bestand die Notwendigkeit, eine eigene europäische Fachsprache des Agrarrechts zu erarbeiten, die sich zwar an die Sprachregelungen der Mitgliedstaaten anlehnt, jedoch nicht die historischen, kulturellen und regionalen Konnotationen der einzelnen mitgliedstaatlichen Rechtsordnungen übernimmt. So wird der Begriff des Landwirts in der EU-Gesetzgebung zwar genannt, zugleich wird aber immer unterstrichen, dass dieser Begriff national ausgelegt werden muss. Soweit die EU ein eigenes Konzept des Landwirts 
zu entwickeln versucht hat, hat sie diese Person sprachlich umschrieben (Betriebsinhaber, aktiver Landwirt). Diese EU-Fachsprache hat daher in den mitgliedstaatlichen Agrarrechtsordnungen nur zu kleinen Veränderungen geführt; im Gegensatz zum materiellen Recht die EU- GAP-Sprache das nationale Agrarrecht nicht harmonisiert. Dadurch ist neben der nationalen Agrarrechtssprache jeweils noch eine eigene EU-GAP-Fachsprache entstanden. Zugleich hat aber die EU durch die Übersetzung nationaler Begrifflichkeiten in EU-Dokumenten, seien es des Gesetzgebers oder des EUGH, durch den EU-Übersetzungsdienst auch wesentlich beigetragen, die sprachliche präzise Übersetzung nationaler Fachbegriffe zu ermöglichen und damit ein Rechtsvergleichung wesentlich zu fördern. Diese Parallelität der europäischen Rechtssprache und der jeweiligen nationalen Sprachen ist keine Besonderheit des Agrarrechts, sondern Ausdruck der besonderen Sprachenpolitik der EU.

Denn die Europäische Union hat die positiven Wirkungen einer Vielfalt von Muttersprachen früh erkannt ${ }^{1}$. Die allererste Verordnung der damals noch als EWG bezeichneten Union betraf im Jahr die Regelung der Sprachenfrage der Europäischen Wirtschaftsgemeinschaft ${ }^{2}$. Sie ist seitdem infolge des Beitritts neuer Länder zur EU entsprechend mehrfach ergänzt worden und legt, zusammen mit Artikel 55 Absatz 1 EUV, die Amtssprachen der Europäischen Union fest. Was 1957 mit den vier Amtssprachen Französisch, Deutsch, Niederländisch bzw. Flämisch und Italienisch der sechs Gründungsmitglieder begann, hat sich rund sechzig Jahre später zu folgenden 24 EU-Amtssprachen in den (noch) 28 EU-Staaten entwickelt: Englisch, Deutsch, Französisch, Italienisch, Spanisch, Portugiesisch, Gälisch (Irisch), Griechisch, Niederländisch, Dänisch, Schwedisch, Finnisch, Estnisch, Litauisch, Lettisch, Polnisch, Slowakisch, Slowenisch, Tschechisch, Ungarisch, Bulgarisch, Rumänisch, Maltesisch und Kroatisch. Einzig Luxemburg hat auf die Erklärung seiner Landessprache, des Letzeburgischen, zur EU-Amtssprache verzichtet. Diese gleichwertige Behandlung aller mitgliedstaatlichen Landessprachen als Amtssprachen ist weltweit einzigartig. Sie wertet die Sprachen kleinerer Länder auf, die bei Zugrundelegung eines rein demographischen Kriteriums unberücksichtigt geblieben wären. So ist die meistgesprochene Muttersprache der Union das Deutsche, es folgen Italienisch, Englisch, Französisch, Spanisch und Polnisch. Insgesamt ist die meistgesprochene bzw. meisterlernte Sprache allerdings das Englische. Die EU grenzt sich damit bewusst von anderen europäischen internationalen Organisationen ab, die wie der

${ }^{1}$ Das deutsche Bundesverfassungsgericht spricht davon, dass die Sprachenvielfalt innerhalb der Europäischen Union angelegt ist, BVerfGE 89, S. 155 (178).

${ }^{2}$ ABl. L 17 vom 6.10.1958, S. 385. 
Europarat oder die NATO nur zwei Amtssprachen (Englisch und Französisch) haben ${ }^{3}$. Zugleich verlässt die EU die historische Tradition in Europa einer Zentralisierung auf eine Verkehrssprache/lingua franca. So wurde die europäische Geschichte vor allem durch drei Verkehrssprachen geprägt: Die lateinische Sprache (abnehmend seit dem späten Mittelalter bis 1867, als Ungarn als letztes Land außer dem Vatikan Latein als Amtssprache aufgibt), Französisch (vom Ende des Dreißigjährigen Krieges 1648 bis zum Versailler Vertrag 1918) und Englisch (seit 1918) .

In konsequenter Fortentwicklung dieser Anerkennung von 24 Amtssprachen erhält jeder Unionsbürger nach Art. 24 AEUV das Recht, sich schriftlich in einer dieser Sprachen an jedes Organ oder an jede Einrichtung der EU zu wenden und eine Antwort in derselben Sprache erhalten ${ }^{5}$.

Diesem Recht des Bürgers steht zugleich auch die Verpflichtung der EU gegenüber, nicht nur die Übersetzung der Anfragen/Antworten zu gewährleisten, sondern auch sämtliche Rechtstexte in alle Amtssprachen zu übertragen. Diese Verpflichtung verlangt organisatorisch den Aufbau einer effizienten und ausreichend ausgestatteten Generaldirektion Übersetzung bei der Europäischen Kommission, einem der größten Übersetzungsdienste der Welt ${ }^{6}$. Daneben verfügen die EU-Organe Parlament und Gerichtshof noch über eigenständige Übersetzungsdienste. So sind allein beim Übersetzungsdienst des Gerichtshofs 600 Juristen beschäftigt ${ }^{7}$.

Diese quantitativ einzigartig hohe Zahl von Beschäftigten im Übersetzungsdienst muss zugleich qualitativ abgesichert sein. Aufgrund der umfassenden Anerkennung als Amtssprachen sind alle übersetzten Rechtstexte der EU, seien sie legislativer, administrativer oder judikativer Art, im rechtlichen Sinne authentisch. Von den Übersetzungsdiensten wird daher eine sprachliche Übertragung verlangt, die dem Willen des erlassenden Organs der EU entspricht. Dieses setzt in der Regel neben den sprachlichen Kenntnissen des Übersetzers auch Rechtskenntnisse voraus.

Diese erhebliche Translationsleistung erfährt zum einen derzeit ihre faktischen Grenzen; insbesondere im Agrarrecht müssen Begriffe aufgrund der besonderen kulturellen und historischen Relativität entweder umschrie-

\footnotetext{
${ }^{3}$ Art. 12 Satzung des Europarates; Art. 14 NATO-Vertrag.

${ }^{4}$ Siehe hierzu umfassend U. Ammon, Verkehrssprachen in Europa - außer Englisch, Tübingen 2001 .

${ }^{5}$ C. Calliess, M. Ruffert, W. Kluth, EUV/AEUV-Kommentar, München 2016, Art. 24 Rn. 3.

${ }^{6}$ EU-Kommission, Übersetzung und Mehrsprachigkeit, http://publications.europa.eu/resource/cellar/e0770e72-afa1-4971-8824-6190512537dc.0007.03/DOC_1 [abgerufen am 30.09.2021].

7 Angaben des EUGH, https://curia.europa.eu/jcms/jcms/Jo2_10741/de/ [abgerufen am 30.09.2021].
} 
ben werden oder es wird der ursprüngliche Begriff in Anführungszeichen verwendet. Die Übersetzungsleistung der EU hat sich jedoch zugleich zu einer unerschöpflichen Quelle für die Agrarrechtssprache und die Agrarrechtsvergleichung entwickelt. Schauen wir uns zunächst die Grenzen an: Diese Grenzen rühren zum einen aus einer trotz großer Ressourcenbindung fortbestehenden Begrenztheit der Mittel der EU für die Übersetzung; zum anderen folgt sie der Tatsache, dass die Zahl der Übersetzer, die etwa einen finnischen agrarrechtlichen Text kompetent ins Maltesische oder ins Portugiesische übertragen können, faktisch begrenzt ist. Daher werden auch nicht sämtliche Texte in alle Amtssprachen übersetzt. Zum Beispiel brauchen interne Vermerke oder Schreiben, die lediglich in einer Sprache versandt werden, nicht übersetzt zu werden. Genauso kann ein Ausschuss beschließen, so lange nur in bestimmten Sprachen zu arbeiten, bis er einen Vorschlag unterbreitet, der auf breiterer Ebene beraten werden und hierzu in allen Amtssprachen vorliegen muss $^{8}$.

Aus Kostengründen wickelt die Kommission, insbesondere die Generaldirektion Landwirtschaft, ihre interne Kommunikation auf Englisch und Französisch ab (und nur noch selten auf Deutsch) ${ }^{9}$. Nur in ihren Beziehungen zu den anderen EU-Institutionen, zu den Mitgliedstaaten und zur Öffentlichkeit macht sie von sämtlichen Amtssprachen Gebrauch. In dem Maße, wie die EU wächst, nehmen auch die praktischen Schwierigkeiten zu, die sich aus der Gleichbehandlung aller Amtssprachen ergeben.

Diese Begrenzungen dürfen jedoch nicht darüber hinwegtäuschen, dass sich durch die umfassende Übersetzungsleistung der EU eine unerschöpfliche Quelle für die Agrarrechtssprache und die Agrarrechtsvergleichung eröffnet hat. Hinsichtlich der Agrarrechtssprache ist eine besondere Wechselwirkung erkennbar. Die Übersetzung hat zum sprachlichen Export von Rechtskonzepten geführt. Hier ist zum Beispiel der Export von Konzepten aus der deutschen Sprache in andere europäische Sprachen erkennbar, da sich die deutsche Sprache für die Entwicklung neuer substantivierter Konzepte in besonderer Weise eignet. Beispiele sind Begriffe wie Direktzahlungen oder Marktorganisation.

Daneben bieten die Übersetzungen der EU und die von ihr in das Agrarrecht aller Mitgliedstaaten übertragenen Rechtskonzepte wie z.B. die ,gute fachliche Praxis“, „Konditionalität“ oder der „Cross-Compliance“ dauerhaft Anlass für rechtsvergleichende Studien.

${ }^{8}$ EU-Kommission, Übersetzung und Mehrsprachigkeit, http://publications.europa.eu/resource/cellar/e0770e72-afa1-4971-8824-6190512537dc.0007.03/DOC_1 [abgerufen am 30.09.2021].

${ }^{9}$ U. Ammon, Die Stellung der deutschen Sprache in der Welt, Berlin u.a. 2015, S. 699ff. 


\section{3. Übersetzung und Rechtsvergleichung in der Kritik}

Dieser positiven Darstellung der Übersetzungsleistungen der EU und der durch sie ausgelösten Rechtsvergleichung steht eine Ansicht gegenüber, die eine Übersetzbarkeit von Rechtskonzepten und in konsequenter Fortsetzung einen die jeweiligen Rechtsordnungen übergreifenden Rechtsvergleich ablehnt. Ausgangspunkt ist die Sapir-Whorf Hypothese, die bezweifelt, dass Übersetzung als kultureller Transformationsakt überhaupt möglich ist und damit im Ergebnis die These von der grundsätzlichen Unübersetzbarkeit fremdsprachiger Texte vertritt ${ }^{10}$. Sie setzt sich aus zwei Thesen zusammen: zunächst aus dem von Pelz formulierten Prinzip der sprachlichen Relativität sowie der Abhängigkeit der Begriffsbildung von der Sprache ${ }^{11}$. Sodann folgt, von einem linguistischen Determinismus ausgehend, daraus eine prinzipielle Unübersetzbarkeit fremdsprachlicher Texte ${ }^{12}$. Die Art und Weise, wie ein Mensch denkt, wird daher nach dieser Vorstellung vorrangig durch die semantische Struktur und den Wortschatz seiner Muttersprache beeinflusst oder bestimmt. Daraus folgt, dass es bestimmte Gedanken einer einzelnen Person in einer Sprache gibt, die von jemandem, der eine andere Sprache spricht, nicht verstanden werden können.

Mit dieser These einher geht die Theorie vom expressivism, die vor allem in den Vereinigten Staaten vertreten wird. Ziel ist, die spezifischen Eigenarten der jeweiligen Rechtsordnung derart zu betonen, dass eine Vergleichbarkeit im Ergebnis nicht mehr angenommen werden kann. Im Ergebnis lehnt damit diese Schule jegliche Rechtsvergleichung $a b^{13}$.

Beide Theorien sind jedoch kritisch zu betrachten. So konnte die Sapir-Whorf Hypothese bislang empirisch nicht belegt werden. Vielmehr ist auch bei bestehendem gleichen sozialen, ökonomischen, kulturellen und historischen Hintergrund Übersetzungen möglich. Hier ist an die Rechtsordnungen wie die Schweiz oder Spanien zu denken, die multilinguale Gesellschaften umfassen. In diesem Fall ist eine binnenstaatliche Übersetzung erforderlich und auch ohne Verständnisverlust möglich. Gleichwohl ist der Hypothese insoweit zuzustimmen, dass eine reine Wortlautübersetzung oft

${ }^{10}$ B.L. Whorf, Sprache - Denken - Wirklichkeit: Beiträge zur Metalinguistik und Sprachphilosophie, Reinbek 2008; siehe kritisch hierzu J. McWhorter, The language hoax: why the world looks the same in any language, Oxford 2014, S. $3 \mathrm{ff}$.

${ }^{11}$ H. Pelz, Linguistik. Eine Einführung, Hamburg 2013; A. Blanco Salgueiro, La relatividad lingüistica, Madrid 2017.

${ }_{12}$ Siehe hierzu G. Deutscher, Im Spiegel der Sprache, warum die Welt in anderen Sprachen anders aussieht, München 2010, S. 148.

${ }_{13}$ Siehe hierzu umfassend M. Tushnet, The Possibilities of Comparative Constitutional Law, „The Yale Law Journal” 1999, Vol. 108, S. 1225, 1269 f. 
den Inhalt der Erklärung nur unzureichend oder gar falsch wiedergibt. Jede Übersetzung muss notwendigerweise den Empfängerhorizont vor Augen haben und sich ihm anpassen, auch wenn dies eine Lösung vom Wortlaut zur Folge hat. Dies kann aber zu erheblichen Schwierigkeiten führen, wenn ein Gesetz übersetzt werden soll, bei dem der Wortlaut im Vordergrund steht. Das Problem kann an folgendem Beispiel verdeutlicht werden:

Der Norminhalt eines Gebotssatzes drückt eine Pflicht in der Form eines Gebots aus. In englischer Sprache wird der normative Operator von Gebotssätzen des materiellen Rechts mit shall ausgedrückt, während das Modalverb must oder die einfache Gegenwartsform in der Regel nur bei der Formulierung von Gebotssätzen des formellen Rechts, also in Verfahrensvorschriften verwendet wird. Der Gebrauch von shall hat sich als Ausdruck des juristischen Imperativs bereits bei der Übersetzung von lateinischen Texten des römischen Rechts ins Englische durchgesetzt. Trotz der langjährigen Übersetzungspraxis in Kanada, das seit 1867 zwei Amtssprachen (Englisch und Französisch) auf Bundesebene kennt, musste der englischsprachige Richter Pigeon seine französisch sprechenden Kollegen wiederholt daran erinnern, dass das englische shall nicht mit dem französischen Futur übersetzt werden darf ${ }^{14}$. Als Übersetzungsrichtlinie empfahl der kanadische Interpretation Act von 1970, das englische shall ins Französische mit dem Modalverb doit oder sogar mit devra zu übersetzen. Im Verlauf der Zeit aber bestanden Übersetzer und Gesetzesredaktoren auf ihrem Recht, Gebotssätze in „natürlichem“ Französischen zu formulieren, d.h. mit der einfachen Gegenwartsform wiederzugeben, wie dies im Code Civil Napoléon praktiziert wurde. Während der kanadischen Gesetzgebungsreform der achtziger Jahre wurde die Übersetzungsrichtlinie dementsprechend geändert und lautet heute wie folgt: „L'obligation s'exprime essentiellement par l'indicatif présent du verbe porteur de sens principal, et, à l'occasion, par des verbes ou expressions comportant cette notion" $" 15$.

Hingegen kann der Theorie des expressivism nicht gefolgt werden. Sie stellt fälschlicherweise auf die unterschiedliche Rechtsordnung ab, die unvergleichbar wäre. Indes muss auf das zugrundeliegende tatsächliche Rechtsproblem, bzw. Regelungsbedürfnis abgestellt werden. Diese Rechtsprobleme sind selbst im lokal, historisch und kulturell derart relativ geregelten Agrarrecht im Grundsatz vergleichbar. So mag das landwirtschaftlich Erbrecht regional verschieden sein und historisch gewachsene Unterschiede aufweisen. Im Ergebnis gehen aber alle landwirtschaftlichen Erbrechtssysteme Europas auf einige wenige Grundmodelle zurück, die

${ }^{14}$ Nach S. Šarčević, Das Übersetzen normativer Rechtstexte, in: P. Sandrini (Hrsg.), Übersetzen von Rechtstexten, Tübingen 1999, S. 109.

${ }_{15}$ Art. 11 Loi d'interprétation, LRC 1985, c I-21. 
entweder autochthon enstanden sind oder durch Rezeption übertragen worden sind: Die Realteilung vs. Alleinerbensystem, das Jüngstenrecht vs. das Ältestenrecht, die Privilegierung des Hoferbes vs. die Gleichbehandlung aller Hoferben, die Privilegierung des landwirtschaftlichen Besitzes vs. die einheitliche Betriebsübergabe.

Übersetzungen und Rechtsvergleichungen sind des Weiteren nicht Modeerscheinungen einer globalisierten Welt des XX. und XXI. Jahrhunderts. Die EU führt im Grunde nur eine in Europa seit Jahrhunderten bestehende Tradition einer binnenstaatlichen sowie auch externen Übersetzung fort, an die eine ebenso lange Geschichte der Rechtsvergleichung, auch im Agrarrecht, anknüpft. Diese soll im Folgenden dargelegt werden.

\section{Binnenstaatliche Übersetzungen}

Ein binnenstaatlicher Übersetzungsbedarf besteht zweifelslos in multilingualen Gesellschaften mit mehreren Amtssprachen wie z.B. die Schweiz oder Belgien. Bereits aus der Rechtsnatur als Amtssprache folgt die Verpflichtung des Staates zur Übersetzung der staatlichen Rechtstexte. Zudem kann nur durch eine entsprechende Übersetzung die Funktion des Rechts, einheitlich und umfassend zu wirken, erreicht werden.

Jedoch hat die binnenstaatliche Übersetzung geschichtlich eine andere Wurzel. Die binnenstaatliche Übersetzung setzt geschichtlich in den Fällen ein, in denen die Fachsprache in einer ,fremden" Sprache, in der Regel Latein, verfasst war, die somit nur für einen begrenzten Kreis gebildeter Juristen verständlich war. Der Bürger als Adressat wurde durch die Ge- und Verbote des Rechts nicht unmittelbar erreicht. Dies gelang erst durch die Übersetzung in die Landessprache. Vor allem in ländlichen Räumen und bei landwirtschaftlichen Rechtsproblemen bestand ein besonderer Bedarf an landessprachlichen Regelungen, da die Kenntnisse des Lateins gering waren und sich zahlreiche agrarrechtliche Regeln gewohnheitsrechtlich in der Sprache der jeweiligen Region gebildet hatten.

Es verwundert daher nicht, dass sich erstmalig findet sich ein auf römischen Rechtsinhalten basierender und damit ,übersetzter" landessprachlicher Rechtstext im provenzalischen Kodex-Summa Lo Codi aus der Mitte des XII. Jhd. Die Summa hat ihren Platz zwischen vorhandener volkssprachlicher und gelehrter lateinischer Tradition. Das Provenzalische bzw. Okzitanische war die erste romanische Literatursprache, in der bereits in der ersten Hälfte des zwölften Jahrhunderts Rechtsurkunden in größerer Zahl verfasst wurden. Es gab damit bereits einen gewissen Ansatz für eine eigenständige 
Fachsprache, auch wenn diese keinen Amtscharakter hatte. Das Werk Lo Codi weicht jedoch inhaltlich und sprachlich stark von seinen lateinischen Vorlagen ab, aus der er weite Teile seines Inhalts übernimmt. Im Gegensatz zur lateinischen Vorlage ist Lo Codi erklärender und didaktischer. Es wird vermutet, dass diese textlichen und sprachlichen Differenzen auf ein Publikum ausgerichtet waren, das ländlich geprägt und damit nicht juristisch gebildet war, sondern ein volkssprachliches Kompendium des anwendbaren Rechts für die Anwendung brauchte. Bei den Verfassern muss es sich hingegen um Personen gehandelt haben, die im römischen Recht gebildet worden waren. Sie schufen daher den vereinfachten und pädagogisch aufbereiteten Text nicht für sich selbst, sondern für ein anderes Publikum ${ }^{16}$.

In einer Traditionslinie zu Lo Codi liegen die Rechtstexte (Partidas) Alfons X (genannt der Weise) von Kastilien (Spanien), die in der Zeit von 1256 und 1265 in kastilischer Sprache (heutiges Spanisch) verfasst wurden. Es handelt sich hierbei um eine Kodifizierung des bestehenden Rechts, insbesondere auch des Agrarrechts, das insbesondere auf dem in lateinischer Sprache verfassten Corpus Iuris Civilis, dem kanonischen Recht und anderen Rechtstexten beruht sowie zusätzlich noch biblische und philosophische Textauszüge, insbesondere von Aristoteles, Seneca, Boethius sowie von Thomas von Aquin, enthält. Ziel war die Schaffung eines für die Bevölkerung verständlichen Rechtstextes, um die Durchsetzung des Rechts zu vereinheitlichen und zu sichern ${ }^{17}$.

Dieses Ziel verfolgten auch die Statute des englischen Königs Edward III aus dem Jahr 1362. Entgegen der vormaligen normannischen Tradition waren diese Rechtstexte nicht ausschließlich auf Französisch verfasst und damit nur für die gelehrten Schichten verständlich (das sog. law french), sondern erstmalig auch in der Landessprache Englisch ${ }^{18}$.

Die binnenstaatliche Übersetzung entfällt grundsätzlich mit der Gleichsetzung der Fachsprache und der Volkssprache. Dies erfolgt zum ersten Mal in Deutschland mit dem Sachsenspiegel, dem von Eike von Repgow in den Jahren zwischen 1220 und 1235 entstandenen Werk, das gemeinsam mit dem Mühlhäuser Reichsrechtsbuch ${ }^{19}$ das älteste Rechtsbuch des deutschen Mittelalters ist.

${ }^{16}$ Hierzu umfassend F. Derrer, Lo Codi. Eine Summa codicis in provenzalischer Sprache aus dem XII. Jahrhundert. Die provenzalische Fassung d. Handschrift A (Sorbonne 632), Zürich 1974.

${ }_{17}$ M. Scheppach, Las siete Partidas, Entstehungs- und Wirkungsgeschichte, Pfaffenweiler 1991.

${ }^{18}$ H.G. Richardson, G.O. Sayles, The English Parliament in the Middle Ages, London 1981.

${ }^{19}$ H. Lück, Mühlhäuser Reichsrechtsbuch, in: G. Alper, M. Puhle (Hrsg.), Aufbruch in die Gotik, Mainz 2009, S. 379-381. 
Der Sachsenspiegel ist das erste umfangreiche Rechtsbuch nicht in Latein, sondern in niederdeutscher Sprache. Der Sachsenspiegel war zwar kein Gesetz. Der Autor wollte das überlieferte Recht seines Stammes und das Recht als Bestandteil der christlichen Weltordnung schriftlich niederlegen. Ein großer Teil des Werkes widmet sich agrarrechtlichen Fragen. Eike von Repgow schuf höchstens unbewusst neues Recht und hielt auch an nicht mehr gängigem Recht fest. Aus diesem Traditionalismus gewann das Rechtsbuch seine Autorität, sodass es schon bald als ein offizielles Gesetzbuch betrachtet wurde $^{20}$.

Diese Korrelation zwischen Fachsprache und Landessprache ist nunmehr weitgehend überwunden. Sie verbleibt noch in zwei unterschiedlichen Bereichen.

1. Soweit Völkerrecht in nationales Recht in dualistischen Systemen wie Deutschland über einen innerstaatlichen Rechtsanwendungsbefehl integriert wird, bedarf es einer Übersetzung der internationalen Vereinbarung ${ }^{21}$. Soweit diese nicht ausdrücklich als authentische Vertragssprache anerkannt ist, ist die Übersetzung nur ein sprachliches Hilfsmittel für die Rechtsadressaten.

2. Die juristische Fachsprache weicht in vielen Punkten vom allgemeinen Sprachverständnis ab. Diese Abweichung findet graduell unterschiedlich statt, hier überlagern sich mehrere Ebenen ${ }^{22}: \mathrm{Zu}$ diesen Ebenen gehört die Rechtsetzung mit ihrer Gesetzessprache und anderen Texten (Verträge, Satzungen), das Rechtswesen mit der Rechtspflege und -anwendung (Urteile, Aussagen, Gutachten, Klageschriften, usw.), die Rechtswissenschaft (Monographien, Aufsätze) und als letzte Ebene die Verwaltung mit der Behördensprache und dem institutionellen Schriftverkehr. Ausgehend von dieser pragmatisch-funktionalen Einteilung können Rechtstexte nach Gesichtspunkten der Fachspezifität und Verständnisschwierigkeit und damit „Übersetzungsbedürftigkeit“" unterschieden werden. So gehören Texte aus dem Bereich der Rechtswissenschaft und Rechtsdogmatik aufgrund ihres Abstraktionsgrades und ihres Adressatenkreises zu den fachspezifischsten und damit zu den ,übersetzungsbedürftigsten“ Texten, während die Behördensprache zwar auf rechtliche Inhalte referiert, sich aber an Laien richtet.

${ }^{20}$ H. Lück, Der Sachsenspiegel: das berühmteste deutsche Rechtsbuch des Mittelalters, Darmstadt 2017.

${ }^{21}$ Th. Maunz, G. Dürig, M. Herdegen, GG-Kommentar, 85. EL November 2018, GGArt. 25 Rn. 4.

22 Vgl. C. Fuchs-Khakhar, Die Verwaltungssprache zwischen dem Anspruch auf Fachsprachlichkeit und Verständlichkeit: ein Vergleich der Darstellungen dieses Konfliktes in der deutschen Verwaltungssprache und der Vorschläge zu einer Bewältigung seit 1958; ergänzt durch einen Blick auf die neueren Ansätze zur Verbesserung der Verwaltungssprache in Großbritannien, Tübingen 1987, S. 36ff. 
Rechtsetzende Texte nehmen eine gewisse Sonderstellung ein, da ihre performative Funktion besondere Probleme der Interpretation und auch der Textproduktion aufwirft ${ }^{23}$. Im Folgenden soll jedoch auf diese spezifische Bedeutung des Übersetzungskonzepts nicht weiter eingegangen werden.

\section{5. Übersetzungen trotz gleicher Amtssprache}

Übersetzungsbedarf besteht jedoch auch bei gleicher Amtssprache, wenn sie in unterschiedlichen nationalen Rechtsordnungen verwendet wird. In diesem Zusammenhang wird die Unterscheidung zwischen der Landessprache und der Fachsprache bedeutsam. Das Recht benutzt eine von der Landessprache abgrenzbare Fachsprache. Ausgangspunkt ist die Feststellung, dass von einer einheitlichen Fachsprache des Rechts höchstens als Abstraktion gesprochen werden kann. Sie setzt sich in concreto zusammen aus den Rechtssprachen der einzelnen nationalen Rechtsordnungen. Es darf nicht z.B. von einer englischen, französischen oder deutschen Rechtssprache ausgegangen werden. Den obersten Kommunikationsrahmen stellt die Rechtsordnung dar, sie beeinflusst nicht nur die rechtlichen Inhalte, sondern auch die Sprache und die Sprachkonventionen. Kommunikation erfolgt damit stets im Rahmen einer bestimmten Rechtsordnung. Die Rechtsordnung bestimmt alle kommunikativen Parameter, von der oder den verwendeten Sprachen über die Begriffe und Benennungen bis hin zu einzelnen Textsortenkonventionen. In diesem Sinne muss etwa im deutschsprachigen Raum zwischen einer Rechtssprache Deutschlands, einer Rechtssprache Österreichs und einer Rechtssprache der Schweiz differenziert werden ${ }^{24}$. So versteht das Schweizer Verfassungsrecht unter dem Begriff Bundesrat ein exekutives Organ, während das deutsche Verfassungsrecht darunter ein legislatives Organ fasst. Spricht der österreichische Jurist von der Execution, so versteht der deutsche Jurist darunter die Hinrichtung als Strafe, nicht hingegen die an sich gemeinte zivilgerichtliche Zwangsvollstreckung. In Deutschland unterscheidet das Recht zwischen Pension (Leistungen für Beamte) und Rente (Leistungen für Arbeitnehmer). Seit 1964 werden in Österreich die Leistungen an Beamte „Ruhegenuss“" genannt, und Arbeitnehmer bekommen eine Pension.

${ }^{23}$ Vgl. J.N. Levi, What is meaning in a legal text? A first dialogue for Law and linguistics, „Washington University Law Quarterly“ 1995, Vol. 73, Issue 3, http://openscholarship.wustl.edu/ law_lawreview/vol73/iss3/2 [abgerufen am 30.09.2021].

${ }^{24}$ Vgl. G. de Groot, Recht, Rechtssprache und Rechtssystem -Betrachtungen über die Problematik der Übersetzung juristischer Texte, „Terminologie \& Traduction“ 1991, Nr. 3, S. 279; K. Paluszek, Die Besonderheiten der österreichischen Rechtssprache, „Comparative Legilinguistics“" 2014, Nr. 19, S. 27. 


\section{6. Übersetzung als Voraussetzung der Rechtsvergleichung}

Die wissenschaftliche Auseinandersetzung mit fremdsprachigen ausländischen Rechtstexten setzt grundsätzlich ein Sprachverständnis durch den Rechtsvergleicher voraus. Dadurch wird der Forschungsbereich der Rechtsvergleichung faktisch bereits erheblich beeinträchtigt, denn die Wissenschaft beschäftigt sich vorrangig mit den Rechtsordnungen, deren Landessprache zu den großen Verkehrssprachen gehört (Englisch, Französisch, Spanisch). Andere Rechtsordnungen indes, wie z.B. das deutsche oder das polnische Recht können aufgrund beschränkter Sprachkenntnisse im Ausland nur noch beschränkt untersucht werden. Will eine faktisch in der rechtsvergleichenden Perspektive unterrepräsentierte Rechtsordnung wie die deutsche oder die polnische daher ihre Rezeption im Ausland fördern, kann sie dies nur durch Übersetzung in eine Verkehrssprache erreichen.

\section{Förderung der Rechtsvergleichung durch Übersetzung}

\subsection{Geschichtlicher Hintergrund}

Auch diese besondere Förderfunktion der Übersetzung zugunsten der Rechtsvergleichung hat eine ebenso lange Tradition in Europa. Ein besonders deutliches Beispiel stammt aus der Epoche der Französischen Revolution. Zwischen November 1792 und Dezember 1793 richtete die Republik ein Übersetzungsbüro in der Convention Nationale ein, dass Übersetzungen der Rechtsakte der Französischen Republik in verschiedene europäische, aber auch außereuropäische Sprachen erstellte (Deutsch, Englisch, Italienisch, Niederländisch, Polnisch, Russisch, Schwedisch, Spanisch und Arabisch). Die Übersetzung zielte darauf, das ,neue“ Recht der jungen Republik in den europäischen und außereuropäischen Ländern bekannt zu machen ${ }^{25}$.

Dabei beschränkte sich die Übersetzung nicht allein auf legislative Akte. Auch Gerichtsurteile wurden übersetzt. Dies war jedoch nur möglich bzw. sinnvoll, weil erst durch die Justizreform der Französischen Revolution vom August 1790, die den Aufbau von Gerichtsurteilen festlegte, das begründete Urteil eingeführt wurde. Als historisches Vorbild diente das römische Formularprozess. Neu war für die französische Rechtsprechung die obligatorische Angabe von Gründen für die Entscheidung. Obwohl es bereits im 13. Jhd.

${ }^{25}$ M. Schreiber, Nationalsprache - Regionalsprache - Nachbarsprache, Zur Übersetzungspolitik während der Französischen Revolution, in: D. Dizdar, A. Gipper, M. Schneider (Hrsg.), Nationenbildung und Übersetzung, Berlin 2015, S. 75 (82). 
erste Ansätze für eine begründete Entscheidung gegeben hatte, setzte sich vom 14. Jhd. bis zum Ende des Ancien Régimes das begründungslose Urteil durch. Einmal etabliert sollte das jugement nonmotivé die gesamte Rechtsprechung des Ancien Régimes beherrschen. Erst seit 1790 finden sich Begründungserwägungen, die eine Übersetzung überhaupt als sinnvoll erweisen lassen ${ }^{26}$.

\subsection{Die Förderung der Rechtsvergleichung durch Übersetzung in der heutigen Zeit}

Aufgrund des erheblichen Ressourcenbedarfs beschränkt sich heute die Übersetzung der Rechtstexte in Deutschland auf ausgewählte Gesetze, denen das Bundesjustizministerium eine besondere grenzüberschreitende Relevanz aus rechtspraktischer sowie aus rechtswissenschaftlicher Sicht zuspricht. Es handelt sich neben dem Grundgesetz um 104 Bundesgesetze und -verordnungen, die ins Englische übersetzt worden $\operatorname{sind}^{27}$. Auf Landesebene finden sich indes keine vergleichbar systematische Übersetzung durch staatliche Stellen. In einem etwas geringerem Umfang, jedoch dafür auf sechs Sprachen erstreckt (Deutsch, Spanisch, Englisch, Italienisch, Chinesisch und Arabisch) bietet Frankreich über das Internetportal Legisfrance Übersetzungen von staatlichen Rechtsakten $a^{28}$.

Bei Gerichtsurteilen bietet das BVerfG regelmäßig Übersetzungen der Urteile von grenzüberschreitender Transzendenz an. Es handelt sich hierbei um durchschnittlich 22 Urteile im Jahr, die ins Englische übersetzt werden ${ }^{29}$. Weitaus umfassender werden die Pressemitteilungen mit einer Kurzzusammenfassung des Urteilstenors und der wesentlichen Gründe übersetzt. Die Urteile der Bundesgerichte werden punktuell übersetzt und auf einer gemeinsamen Plattform der Präsidenten den Obersten mitgliedstaatlichen Gerichte der Europäischen Union veröffentlicht. Diese Plattform läßt vergleichbare Entwicklungen in anderen europäischen Mitgliedstaaten zugunsten einer stärkeren Förderung der grenzüberschreitenden Rezeption der mitgliedstaatlichen Urteile der obersten Gerichte erkennen. Leider muss festgestellt

${ }^{26}$ M. Schreiber, La phrase unique: Die Ein-Satz-Struktur in Texten der Französischen Revolution und deren Übersetzungen, in: W. Dahmen, G. Holtus u.a. (Hrsg.), Sprachvergleich und Übersetzung: Die romanischen Sprachen im Kontrast zum Deutschen, Tübingen 2017, S. 81.

27 Die übersetzten Gesetze sind auf der Seite des BMJ zu finden unter http://www.gesetzeim-internet.de/Teilliste_translations.html [abgerufen am 30.09.2021].

${ }_{28}$ Siehe hierzu das Portal Legisfrance https://www.legifrance.gouv.fr/Traductions.

29 Die Liste der übersetzten Urteile findet sich unter https://www.bundesverfassungsgericht. de/SiteGlobals/Forms/Suche/EN/Entscheidungensuche_Formular.html?language_=en [abgerufen am 30.09.2021]. 
werden, dass die Übersetzung und damit auch die Rezeption ausländischer Gerichtsurteile zum Agrarrecht weit unterdurchschnittlich ist. Hier besteht noch ein erheblicher Nachholbedarf.

\subsection{Stand der Rechtsvergleichung in den Staaten Europas}

Diese durch die Übersetzung geförderte Rezeption ausländischer Rechtstexte und Gerichtsurteile darf nicht darüber hinwegtäuschen, dass der Grad der Rezeption in den Staaten Europas unterschiedlich stark ausgeprägt ist.

Eine umfassende Verpflichtung zur Rechtsvergleichung findet sich in keiner Verfassung eines europäischen Staates. Beschränkt auf die durch Menschenrechtspakte gesetzten internationalen Standards findet sich in Art. 10 Abs. 2 der spanischen Verfassung die Verpflichtung die Normen, die sich auf die in der Verfassung anerkannten Grundrechte und Grundfreiheiten beziehen, in Übereinstimmung mit der Allgemeinen Erklärung der Menschenrechte und den von Spanien ratifizierten internationalen Verträgen und Abkommen über diese Materien auszulegen. Ohne eine derartige Verpflichtung verlangt in Frankreich der Senat für jede Gesetzesänderung eine summarische Untersuchung vergleichbarer Regelungen in Europa. Sie bilden, da sie veröffentlicht werden, ein großes Reservoir für den Rechtsvergleicher. Dieses Verwerten des europäischen Erfahrungspools geht über die Gerichtsbarkeit bis hin zur Verwaltung, wo gerade im Zusammenhang mit der Verwaltungsmodernisierungswelle die Heranziehung ausländischer Modelle eine unverzichtbare Voraussetzung im Entscheidungsfindungsprozess der Verwaltung ist. Leider ist aber auch hier das Agrarrecht noch sehr unterrepräsentiert. So finden sich seit 1998 nur vier rechtsvergleichende Untersuchungen mit agrarrechtlichem Bezug ${ }^{30}$.

In Deutschland bedient sich der Bundestag unregelmäßig vergleichbarer rechtsvergleichender Untersuchungen des Wissenschaftlichen Dienstes. Haben wir aber eine derartige explizite Ermächtigung zur Rechtsvergleichung im deutschen Grundgesetz? Zunächst einmal ist hier auf die Integrationsklausel in Art. 23 Abs. 1 GG zu verweisen. Soweit die Integrationsklausel jedoch über die Rechtsordnung der Union hinausgeht und zur Integration der Rechtsordnungen der Mitgliedstaaten herangezogen wird, setzt das BVerfG dem zu Recht eine Grenze. So weist das Gericht bei Ungleichbehandlungen,

${ }^{30}$ LC 269: La couverture des risques en agriculture et les assurances agricoles (juin 2016); LC 74: Le contrôle de la sécurité alimentaire (mai 2000); LC 71: La chasse (mars 2000); LC 32: Le régime d'administration des ports maritimes (janvier 1998), siehe: https://www.senat.fr/legislationcomparee-theme/lc-agriculture-et-peche.html [abgerufen am 30.09.2021]. 
die wirtschaftliche Konsequenzen hat, ausdrücklich darauf hin, dass es nicht den Vergleichsmaßstab ansetzen kann, da der Gesetzgeber nur innerhalb seines Herrschaftsbereichs an den Gleichheitssatz gebunden ist ${ }^{31}$. Es begründet daher keinen Verstoß gegen Art. 3 Abs. 1 GG, wenn die Bundesrepublik Regelungen erlässt, die von jenen in anderen Mitgliedstaaten der Europäischen Union abweichen ${ }^{32}$.

Die deutsche Rechtsordnung kennt aber eine konkludente Klausel mit Bezug zur Rechtsvergleichung, nämlich das Prinzip der völkerrechtsfreundlichen Auslegung des Grundgesetzes. Dieses Prinzip zwingt dazu, die völkerrechtliche Vertragsnorm im Lichte ihrer völkerrechtlichen Praxis und damit der Verfassungswirklichkeit der Staaten zu prüfen. Über die Transformation des Völkerrechts findet somit das ausländische Recht Eingang in unsere Rechtsordnung. Dabei ist diese Integrationswirkung nicht beschränkt auf das Völkervertragsrecht. So sind nach Art. 38 des IGH-Statuts die Rechtssätze der zivilisierten Nationen eine Quelle des Völkerrechts. Wir müssen aber festhalten: Eine normative Ermächtigung zur unmittelbaren Integration des ausländischen Rechts ist in unserer Verfassung nicht vorgesehen. Wir können insoweit nur von einer verfassungsrechtlichen Ermächtigung zur mittelbaren Rechtsvergleichung über den Weg des Völkerrechts sprechen. Dem steht auch das Konzept des offenen Verfassungsstaates nicht entgegen, da daraus keine neuen Befugnisse entnommen werden können.

Obgleich das Ergebnis zunächst ernüchternd klingt, muss man sich wieder klar machen, dass der Verfassungsgeber, der Gesetzgeber, die Gerichte und die Verwaltung innerhalb ihrer Gestaltungsspielräume und Wertungen rechtsvergleichende Erkenntnisse jederzeit einbringen können. Einen wesentlichen Beitrag leistet jedoch die Wissenschaft. Akademische Leuchttürme wie Professor Budzinowski eröffnen uns durch ihre vertieften rechtsvergleichenden Forschungen des polnischen, deutschen, italienischen und spanischen rechts die Möglichkeit, die ,gespeicherten Erfahrungsweisheiten“33 anderer Völker zu erfassen.

\section{Zusammenfassung}

Die Rechtsvergleichung im Agrarrecht hat in den letzten Jahrzehnten eine wachsende Rolle in der modernen Agrarrechtswirklichkeit eingenommen und ist heute für die agrarrechtliche Forschung unverzichtbar, um das un-

31 Vgl. BVerfGE 10,354 (371).

${ }^{32}$ BVerfG, 1 BvR 2221/03 vom 1.10.2004, Absatz-Nr. 17.

${ }^{33}$ M. Kriele, Recht als gespeicherte Erfahrungsweisheit; Eine „,konservative“ Theorie des Staates, in: B. Kohler-Koch (Hrsg.), Staat und Demokratie in Europa, Opladen 1992, S. 83-92. 
tersuchte Rechtsinstitut umfassend oder innovativ zu behandeln. Eine der größten Herausforderungen der Agrarrechtsvergleichung ist die Sprache, dem Werkzeug des Juristen. Das Agrarrecht hat eine eigene Fachsprache entwickelt, die sehr oft an tradierten Begriffen anknüpft, die kulturell und historisch mit einer bestimmten Region verbunden sind und daher im besonderen $\mathrm{Ma}$ als relativ bewertet werden müssen. Der Vergleich ausländischer Regeln des Agrarrechts setzt daher beim Rechtsvergleicher nicht nur Sprachfertigkeiten voraus, sondern auch entsprechende Kenntnisse des historischen, kulturellen und regionalen Umfelds. Neben den staatlichen Agrarfachsprachen hat sich eine europäische Sprache der Gemeinsamen Agrarpolitik entwickelt, die sich wechselseitigen befruchten. Die Europäische Union beruht auf der Vielfalt der Rechtssprachen als Ausdruck der Vielfalt der mitgliedstaatlichen Rechtskultur, insbesondere im Agrarrecht. Die Relativität der agrarrechtlichen Fachsprache steht einer Rechtsvergleichung nicht im Wege. Sie verlangt vom rechtsvergleichenden Forscher jedoch jenseits der sprachlichen Kenntnisse auch vertiefte kulturelle, soziale und ökonomische Kenntnisse des jeweils anderen Staates. Ein mit diesen Fähigkeiten ausgestatteter Forscher ist in der Lage, Rechtstexte so zu übersetzen, dass sie von Dritten verstanden und richtig eingeordnet werden können. Die Europäische Union investiert erhebliche Ressourcen in diese Übersetzungsleistungen; die Mitgliedstaaten indes haben bislang die Bedeutung der Rechtsvergleichung für die europäische Integration und für die Fortentwicklung des eigenen Rechtssystems, insbesondere im Agrarrecht, nicht ausreichend erkannt. Dieses Defizit wird in weiten Teilen kompensiert durch die Wissenschaft. Akademische Leuchttürme wie Professor Budzinowski eröffnen uns durch ihre vertieften rechtsvergleichenden Forschungen des polnischen, deutschen, italienischen und spanischen rechts die Möglichkeit, die ,gespeicherten Erfahrungsweisheiten“anderer Völker zu erfassen.

\section{BIBLIOGRAPHIE}

Ammon U. (2001), Verkehrssprachen in Europa - außer Englisch, Tübingen.

Ammon U. (2015), Die Stellung der deutschen Sprache in der Welt, Berlin u.a.

Blanco Salgueiro A. (2017), La relatividad lingüistica, Madrid.

Calliess C., Ruffert M. (Hrsg.) (2016), EUV/AEUV-Kommentar, München.

Derrer F. (1974), Lo Codi. Eine Summa codicis in provenzalischer Sprache aus dem XII. Jahrhundert. Die provenzalische Fassung d. Handschrift A (Sorbonne 632), Zürich.

Deutscher G. (2010), Im Spiegel der Sprache, warum die Welt in anderen Sprachen anders aussieht, München.

Fuchs-Khakhar C. (1987), Die Verwaltungssprache zwischen dem Anspruch auf Fachsprachlichkeit und Verständlichkeit: ein Vergleich der Darstellungen dieses Konfliktes in der deutschen Verwaltungssprache und der Vorschläge zu einer Bewältigung seit 
1958; ergänzt durch einen Blick auf die neueren Ansätze zur Verbesserung der Verwaltungssprache in Großbritannien, Tübingen.

Groot G. de (1991), Recht, Rechtssprache und Rechtssystem -Betrachtungen über die Problematik der Übersetzung juristischer Texte, „Terminologie \& Traduction“ Nr. 3.

Kriele M. (1992), Recht als gespeicherte Erfahrungsweisheit; Eine „,conservative“ Theorie des Staates, in: B. Kohler-Koch (Hrsg.), Staat und Demokratie in Europa, Opladen.

Levi J.N. (1995), What is meaning in a legal text? A first dialogue for Law and linguistics, „Washington University Law Quarterly” Vol. 73, Issue 3, http://openscholarship.wustl. edu/law_lawreview/vol73/iss3/2 [abgerufen am 13.03.2019].

Lück H. (2009), Mühlhäuser Reichsrechtsbuch, in: G. Alper, M. Puhle (Hrsg.), Aufbruch in die Gotik, Mainz.

Lück H. (2017), Der Sachsenspiegel: das berühmteste deutsche Rechtsbuch des Mittelalters, Darmstadt.

Maunz Th., Dürig G., Herdegen M. (2018), GG-Kommentar, 85. EL November, München.

McWhorter J. (2014), The language hoax: why the world looks the same in any language, Oxford.

Paluszek K. (2014), Die Besonderheiten der österreichischen Rechtssprache, „Comparative Legilinguistics" Nr. 19.

Pelz H. (2013), Linguistik. Eine Einführung, Hamburg.

Richardson H.G., Sayles G.O. (1981), The English Parliament in the Middle Ages, London.

Šarčević S. (1999), Das Übersetzen normativer Rechtstexte, in: P. Sandrini (Hrsg.), Übersetzen von Rechtstexten, Tübingen.

Scheppach M. (1991), Las siete Partidas, Entstehungs- und Wirkungsgeschichte, Pfaffenweiler.

Schreiber M. (2015), Nationalsprache-Regionalsprache-Nachbarsprache, Zur Übersetzungspolitik während der Französischen Revolution, in: D. Dizdar, A. Gipper, M. Schneider (Hrsg.), Nationenbildung und Übersetzung, Berlin.

Schreiber M. (2017), La phrase unique: Die Ein-Satz-Struktur in Texten der Französischen Revolution und deren Übersetzungen, in: W. Dahmen, G. Holtus u.a. (Hrsg.), Sprachvergleich und Übersetzung: Die romanischen Sprachen im Kontrast zum Deutschen, Tübingen.

Tushnet M. (1999), The Possibilities of Comparative Constitutional Law, „The Yale Law Journal" Vol. 108.

Whorf B.L. (2008), Sprache - Denken - Wirklichkeit: Beiträge zur Metalinguistik und Sprachphilosophie, Reinbek.

\title{
LINGUISTIC CHALLENGES FOR AGRICULTURAL LAW COMPARATIVISM
}

\author{
Sum mary
}

Agricultural law combines two opposing directions of development of law in general. One is connected with the local or regional element and indeed, like no other discipline, is linked to a specific region, culture, tradition and linguistic specificity, being a consequence of the specific nature of agricultural law. On the other hand, at the level of the European 
Union it is characterised by the greatest degree of integration. The Common Agricultural Policy has made agricultural law locally and regionally rooted but, at the same time, it has created a specific technical language of European agricultural law. Therefore, conducting a comparative legal research into the field of agricultural law faces a particular challenge as it requires combining both local and regional linguistic specificities with the European technical language of agricultural law. This relativism of the technical language of agricultural law does not, however, stand in the way of legal comparativism. However, apart from the linguistic knowledge, it requires the researcher to possess thorough cultural, social and economic knowledge of another country. Professor Budzinowski, unlike many other scholars, embodies these skills in his comparative legal research.

Keywords: agricultural law studies, legal comparativism, language, Common Agricultural Policy, relativism

\section{SFIDE LINGUISTICHE POSTE AL DIRITTO AGRARIO COMPARATO}

\section{Riassunto}

Il diritto agrario combina due direzioni opposte di sviluppo del diritto in generale. Da un lato, si tratta di un aspetto locale o regionale. Infatti, come nessun'altra disciplina, essa è legata a una specificità regionale, culturale, tradizionale e infine linguistica. Ciò consegue dalla sua particolare natura. Dall'altro, il diritto agrario è quell'area del diritto a livello dell'Unione europea che si caratterizza per il maggior grado di integrazione. La politica agricola comune è riuscita a far europeizzare questo ambito del diritto, radicato a livello locale e regionale, e al tempo stesso ha fatto nascere un linguaggio tecnico specifico del diritto agrario europeo. Quindi, le ricerche giuridiche comparate di diritto agrario si trovano costrette a dover affrontare una sfida del tutto particolare, vale a dire devono saper combinare le specificità linguistiche, locali e regionali, con il linguaggio tecnico europeo del diritto agrario. Fortunatamente, il relativismo del linguaggio tecnico del diritto agrario non va a ostacolare gli studi giuridici comparati, anche se, oltre alle conoscenze linguistiche, richiede al ricercatore pure una approfondita conoscenza culturale, sociale ed economica di un altro Paese. Il Professor Budzinowski incarna queste abilità nella sua ricerca giuridica comparata come quasi nessun altro scienziato.

Parole chiave: scienza del diritto agrario, studi giuridici comparati, linguaggio, politica agricola comune, relativismo 